\title{
GENERATION OF MULTITEMPORAL THERMAL ORTHOPHOTOS FROM UAV DATA
}

\author{
K. Pech ${ }^{\text {a, } *, \text { N. Stelling }}{ }^{\text {a }}$, P. Karrasch ${ }^{\text {a }}$, H.-G. Maas ${ }^{\text {a }}$ \\ ${ }^{a}$ Technische Universität Dresden, Institute of Photogrammetry and Remote Sensing, 01062 Dresden, Germany - \\ (Katharina.Pech, Nadine.Stelling, Pierre.Karrasch, Hans-Gerd.Maas)@tu-dresden.de
}

Commission I, ICWG I/5

KEY WORDS: Environment, Urban, UAV, thermal camera, multitemporal orthoimage, SIFT

\begin{abstract}
:
The paper deals with using a TIR camera on an UAV for acquiring multitemporal thermal images of a building block against the background of detecting, monitoring and analysing urban heat islands. It is motivated by a research project called EO2HEAVEN (Earth Observation and Environmental Modelling for the Mitigation of Health Risks) which analyses the influence of environmental effects to human health. Therefore, the aim is the generation of thermal orthophotos from UAV data which can be used for further thematic analysis. The paper describes the data acquisition on the one hand and the processing of the obtained data on the other hand. The data acquisition comprises three image flights at different times of day from which only the first two missions could be processed until now. The low image contrasts, the radiometric differences between images as well as the poor initial positioning and orientation values limit the suitability of available software for automatic tie point measurement so that this step was outsourced and implemented in $\mathrm{C}++$. The following aerial triangulation and orthophoto generation was realised in TerraPhoto (Terrasolid). However, two orthophotos could be generated with a geometric resolution of $15 \mathrm{~cm}$. Furthermore, the radiation temperatures from the thermal images were compared to ground measurements to check the correctness of the camera measurements.
\end{abstract}

\section{INTRODUCTION}

In the recent past thermal cameras came into photogrammetric focus. In Luhmann et al. (2011) the increasing importance of the geometrical characteristics of thermal cameras at increasing resolutions is mentioned. The geometric information is essential especially for the generation of thermal orthophotos. Thermal orthophotos are mainly required for spatial analysis of temperature distributions. Recorded at various times and from the air, they can be a valuable contribution to the study of micro Urban Heat Islands (UHI). That means that the climate of an urban area stands out clearly against the surrounding area. This can be for example poorly vegetated parking lots, non-reflective roofs or asphalt roads. This phenomenon is particularly evident on hot windless summer days. Within the project EO2Heaven (Earth Observation and Environmental Modelling for the Mitigation of Health Risks) a study was conducted which evaluated the use of a thermal camera on an UAV for this purpose. While thermal images obtained from satellites or planes have become standard, the UAV as recording platform for thermal cameras is relatively new. Problems like the more instable and susceptible platform to interferences (e.g. blurring caused by wind or rotor movement) compared to airplanes, the smaller and mostly more inaccurate sensors due to weight limitation as well as the smaller image format and the special character of thermal images complicate the processing and represent a challenge in planning. In Hartmann et al. (2012) an automatic orientation of thermal image blocks acquired from an UAV is presented using artificial ground control points. But since a high number of control points is needed, it is not desirable for the practical application of larger areas.
Instead, other ways should be found to support the orientation process. One possibility is to increase the accuracy of the navigation data by using dGNSS for direct georeferencing. In this context, Bláha et al. (2011) analysed the potential of the positioning accuracy of the Falcon 8. Another approach uses UAV-based video sequences and an existing digital landscape model for an integrated georeferencing (Eugster et al., 2007). Thus, on one side a video can improve the orientation parameters, but it can also support the generation of thermal orthophotos by providing additional information, like digital terrain models, digital surface models and control points.

The aim of this paper is the generation of multitemporal thermal orthophotos for climate studies by using thermal images acquired with an entry-level infrared camera on an UAV. In contrast to Hartmann et al. (2012) our application is challenging concerning a five times larger test area with urban character thereby combined with less ground control points (only marginal configuration). Concerning this, section 2 describes the part of data acquisition including the description of the used unmanned aerial system (UAS) and cameras as well as the introduction of the flight planning and realisation. The data processing is the topic of the next section. An important part of the data processing is the extraction of tie points. For this purpose, the approach, presented in Meierhold et al. (2010) using SIFT and fundamental matrix fitting for feature detection and matching between RGB images and intensity images derived from laser scanner data, was adapted. Finally, the results are shown (section 4) and the paper is closed with a summary and an outlook (section 5).

\footnotetext{
* Corresponding author.
} 


\section{DATA ACQUISITION}

\subsection{Unmanned aerial system}

When choosing a suitable receiving platform, also the selection of the sensor equipment - in our case the thermographic camera - plays an important role. Besides the payload, the radiometric characteristics should not be neglected, especially when measuring absolute temperatures. There are thermal cameras for many applications, but only a few comply with the requirements for mobile use with drones. At the time of the flight most lightweights were only imaging cameras. That means, they are well suited for a qualitative observation of objects but not for measuring absolute temperatures. Currently new camera models are in development (calibrated, light and small) which are then suitable for determining areal temperatures from a small drone (e.g. http://www.flir.com/cs/emea/en/view/?id=56345 (20.05.2013)).

For our study the infrared camera InfraTec mobileIR E9 was chosen. With a weight of $980 \mathrm{~g}$, it is not really light-weight and therefore for small drones not feasible. For this reason, the octocopter called HORUS (Hovering Remote controlled Ultralight Sensor platform) was used which was developed by the Fraunhofer Institute for Transportation and Infrastructure Systems IVI. The vehicle has a dimension of $1300 \mathrm{~mm} \mathrm{x}$ $1000 \mathrm{~mm} \times 80 \mathrm{~mm}$ and weighs $1800 \mathrm{~g}$. With a maximum flying time of $40 \mathrm{~min}$ (without payload and under best possible conditions), a maximum payload capacity of $3500 \mathrm{~g}$ and the possibility of autonomous way point navigation it is well suited for photogrammetric applications.

To ensure a sufficient flying time the small and light RGB camera GoPro HD Hero (Table 1) was used as second camera system. Figure 1 shows the flying UAV with the equipped cameras.

\begin{tabular}{|l|l|l|}
\hline Camera & InfraTec mobileIR E9 & GoPro HD Hero \\
\hline Type & $\begin{array}{l}\text { Infrared camera for mobile } \\
\text { use; accuracy: } \\
\pm 2 \mathrm{~K}(0 \ldots 100){ }^{\circ} \mathrm{C} ; \\
\pm 2 \%(<0 \text { and }>100){ }^{\circ} \mathrm{C}\end{array}$ & $\begin{array}{l}\text { Small waterproof } \\
\text { and shockproof } \\
\text { camera } \\
\left(127^{\circ} \text { wide angle }\right)\end{array}$ \\
\hline Image size & $384 \times 288 \mathrm{px}$ & $1920 \mathrm{x} 1080 \mathrm{px}$ \\
\hline Pixel size & $25 \mu \mathrm{m}$ & $2.2 \mu \mathrm{m}$ \\
\hline Focal length & $25 \mathrm{~mm}$ & $\begin{array}{l}\text { estimated } \\
2.1446 \mathrm{~mm}\end{array}$ \\
\hline Frame rate & approximately $20 \mathrm{~Hz}$ & $25 \mathrm{~Hz}$ \\
\hline Weight & $980 \mathrm{~g}$ & $167 \mathrm{~g}$ \\
\hline
\end{tabular}

Table 1. Camera specifications from manufacturer

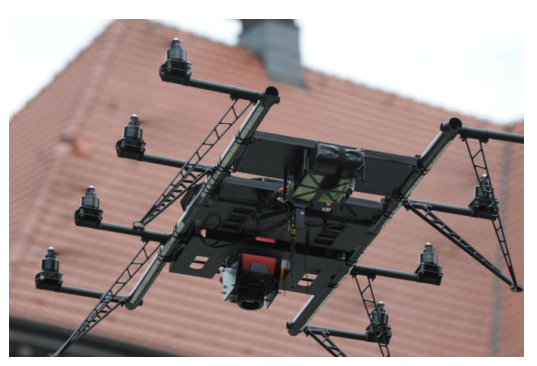

Figure 1. Horus octocopter equipped with thermal infrared camera InfraTec mobileIR E9 and RGB camera GoPro HD Hero
Furthermore, the unmanned aerial system (UAS) consists of a ground station with a computer for flight planning and monitoring, a remote control to transfer mission parameters and an antenna module to receive data from UAV. Besides operator intervention, these components allow autonomous waypoint navigation.

\subsection{Flight planning}

A conventional flight planning was done considering the specifications of the TIR camera and the UAS as well as the characteristics of the test area. The chosen test area, representing a part of the TU Dresden campus, has a size of $260 \times 200 \mathrm{~m}^{2}$ and has an urban character containing different kinds of buildings, streets and vegetation.

An intended geometrical resolution of $15 \mathrm{~cm}$ and a strip overlap of $60 \%$ perpendicular to flight direction lead to a flight altitude of $150 \mathrm{~m}$ and necessitated eight strips to cover the whole test area. Additionally, to stabilise the image block, one cross strip was planned through the centre of the test area. Due to the frame rate of the camera and a flight velocity of $4 \mathrm{~m} / \mathrm{s}$, the longitudinal overlap is of approximately $99 \%$ and a continuous flight without break points was planned.

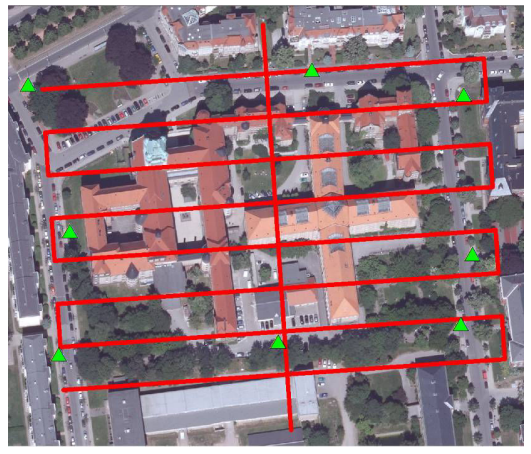

Figure 2. Planned flight path (red line) and positions of control points (green triangles)

orthophoto data source: ATKIS ${ }^{\circledR}$-DOP $\odot$ Staatsbetrieb

Geobasisinformation und Vermessung Sachsen 2013

Around the test area, eight control points were arranged and their positions were determined by dGPS. To realise the visibility in the thermal image data from $150 \mathrm{~m}$ height, the control points were designed using a $1 \times 1 \mathrm{~m}^{2}$ flake board. Due to the characteristics of thermal radiation and the resulting low image contrast, it was important to find materials which differ significantly from there surrounding area. This decision has to be made based on the emission or reflection characteristics in the thermal spectral range. Here, the emissivity is one of the most important features of a specific material. It is notable that metallic objects have a considerably lower emission coefficient than other materials. A combination of two materials with high different emission coefficients can perform high contrast control points. For this reason crinkled aluminium foil was chosen. The centre is marked with a black paper circle of $45 \mathrm{~cm}$ diameter (Figure 3). The positions of the control points can be seen in Figure 2. 


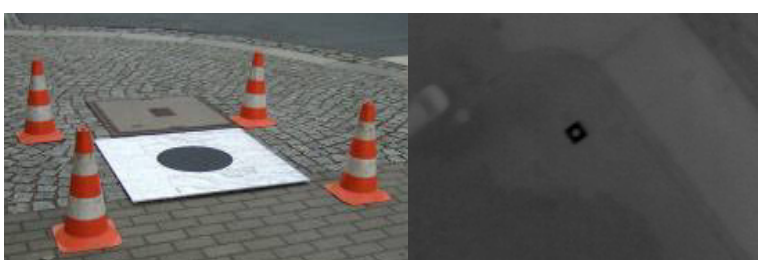

Figure 3. Control points (left: RGB image; right: thermal image from $150 \mathrm{~m}$ height)

\subsection{Realisation}

To consider diurnal temperature variations, three flight missions were carried out at one summer day: one in the morning ( $7 \mathrm{am})$, one at noon $(2 \mathrm{pm})$ and one in the evening $(9 \mathrm{pm})$. As the test area is part of the campus, it was important to choose a weekend day to ensure that there are no people within the test area during the flights. The flights were carried out on 30.06.2012 which was a typical summer day in Germany. The effective flying time of the UAV was decreased to approximately 10 min for safety reasons and considering the weather conditions as well as the payload of about $1147 \mathrm{~g}$. Hence, the planned flight path was divided into two parts to enable battery changing in between. However, the battery service life of the cameras was no problem. They can run for several hours.

Finally, for each flight mission, two thermal videos and two RGB videos were obtained. Additionally, the registered flight trajectories from GPS and IMU sensors were saved in GPX files with a rate of $1 \mathrm{~Hz}$.

\section{DATA PROCESSING}

\subsection{Data checking}

First of all, the acquired data was checked for:

- The compliance of the flight parameter (anomalies in the flight path, height, speed),

- The quality and completeness of the videos itself

- The visibility of control points within the videos

In this process, it was obvious that the frame rate of the thermal camera differs from the information of the manufacturer. The frames were recorded with approximately $12.4 \mathrm{~Hz}$ instead of $20 \mathrm{~Hz}$. This fact is of particular importance for the step of data synchronisation.

Furthermore, the analysis showed variations for each flight parameter which have to be considered during the subsequent processing. Figures 4 and 5 show exemplary the logged speed of the flight mission one in the morning. The flight strips are clearly visible in part one. But it can also be seen that not every flight strip has the same speed. A trend for the forward and backward movement of the UAV is apparent. Part two has more variations than part one. Due to this, each flight strip has to be considered separately for the calculation of the longitudinal overlaps.

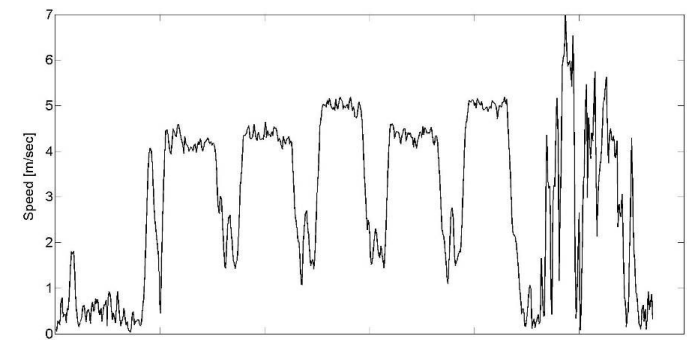

Figure 4. Speed of flight mission one (part one)

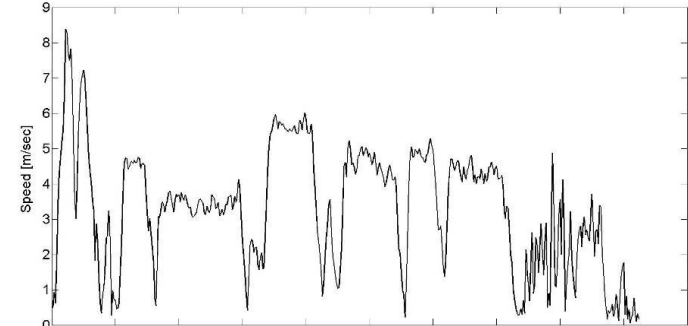

Figure 5. Speed of flight mission one (part two)

The aim of the following data preparation is to achieve a manageable number of images (thermal and RGB data) with approximate positions from the recorded flight trajectory. These data can then act as input for image block orientation by aerotriangulation and successively for the generation of digital terrain models and thermal orthophotos.

\subsection{Data preparation}

3.2.1 Synchronisation: Each video has a length of 9 to 12 minutes (including launch and landing). At first, the binarycoded thermal videos were converted into 16 bit images. There were more than 14000 images extracted for each mission. Because the frame rates of the cameras are higher than the frequency of the registered GPS positions, the GPS positions have to be linear interpolated based on the timestamp and trajectory. Due to a missing common timestamp, the synchronisation of the thermal videos required some user interaction. Because the launch and the landing are not apparent from the trajectory data, an iterative process had to be carried out. At this, the first image of the first flight strip was defined manually and was assigned to the automatically extracted first GPS position (see 3.2.2). Considering an approximate frame rate of $12.4 \mathrm{~Hz}$ of the thermal camera, each following image was assigned an exterior orientation.

3.2.2 Extraction of single flight strips: The beginning of each strip was determined by comparing the calculated course angle (based on the GPS positions) and the planned course angle (known from flight planning). Two additional conditions were introduced

- A certain altitude with a defined threshold value

- Viewing direction of the UAV

These two conditions ensure that the launch and landing are reliably removed. Additionally, the automatic extraction of the flight strips was controlled visually and statistically.

3.2.3 Reduction of image data: The determined averaged height and averaged speed of each strip as well as the desired longitudinal overlap (80\%) and camera parameters serve as input for the subsequent calculation of the image distances. Figure 6 shows the approximate positions of the resulting thermal images (about 180) for mission one. For processing reasons, flight strip one was removed. The area is sufficiently covered by the remaining strips. 


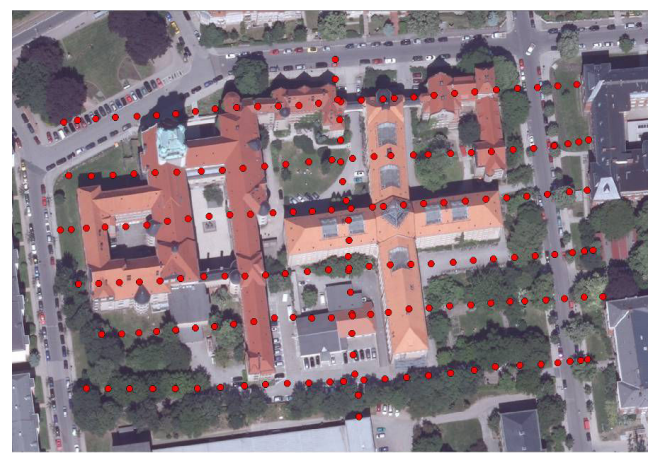

Figure 6. Approximate positions of the resulting thermal images of mission one (part one and two)

orthophoto data source: ATKIS ${ }^{\circledR}$-DOP ๑ Staatsbetrieb

Geobasisinformation und Vermessung Sachsen 2013

\subsection{Tie point extraction}

3.3.1 General: The step of tie point detection and extraction was implemented in $\mathrm{C}++$ because the results of available software like Leica Photogrammetry Suite V9.x, TerraPhoto (Terrasolid), Pix4UAV (Pix4D) and Agisoft PhotoScan (Standard edition V 0.9.0) were non-satisfying.

The Scale-Invariant-Feature-Transform (SIFT) by Lowe (2004) is designed to match corresponding points in images taken from widely different viewpoints. Linear brightness alterations, rotations and translations have no effect on their ability to find and uniquely identify point matches between images. These characteristics allow the feature extraction from thermal images acquired from an UAV platform which show radiometric differences as well as rotation, translation and scale differences e.g. caused by a more instable platform to interferences like wind or by lower positioning accuracy.

3.3.2 Feature detection: For the detection and matching of feature points from the thermal images the SIFT implementation of the OpenCV library V2.4 was used. During the feature detection, the peak threshold which is responsible for discarding low contrast points and the edge threshold for discarding edge responses were adapted to the special data input. Due to the low image contrast of the thermal images, this threshold had to be reduced. The edge threshold ensures that more stable, less edge located keypoints are detected. To reduce the number of instable keypoints the default threshold was also decreased.

3.3.3 Feature matching and outlier reduction: After feature matching using the SIFT descriptor, there are a lot of false matches which would disturb the next step of image orientation. Thus, the following methods were applied to reduce the number of false matches:

- Symmetry criterion

- Fundamental matrix fitting with RANSAC

- Angle criterion

The symmetry criterion keeps only these matches which are found in both matching directions (matching image 1 with image 2 and image 2 with image 1). This ensures more stable tie points. The remaining matches were used for fundamental matrix fitting with RANSAC (Fischler \& Bolles, 1981) to find the set of matches which fulfil the relative orientation of the two overlapping images.

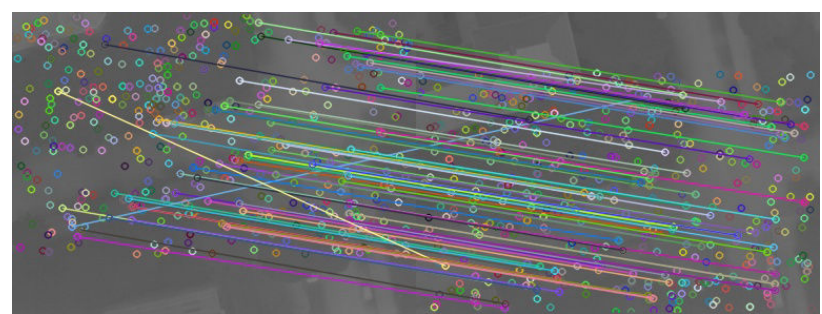

Figure 7. Remaining feature matches after fundamental matrix fitting (image 1 left, image 2 right)

Like seen from Figure 7, there are still some falsely matched keypoints which differ from the correct matches by its orientation. These outliers remain because they are close to the respective epipolar line and therefore comply with the fitted fundamental matrix. By reducing the parameter for epipolar line distance the false matches could not be removed. Thus, in the last step the orientation of the matches is analysed using a histogram analysis to find the most frequent occurrence. This orientation is used to discard deviant matches (Figure 8).

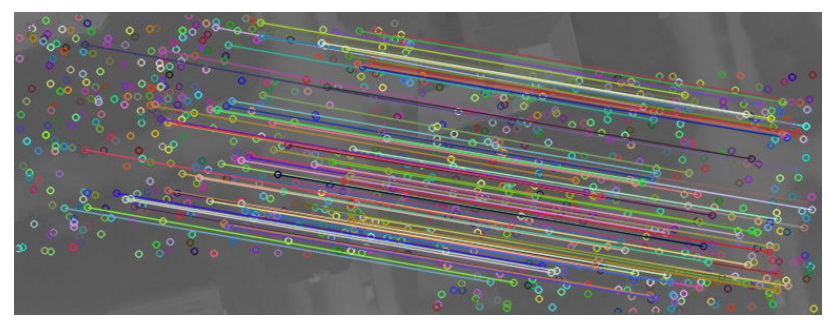

Figure 8. Final tie points detected from two overlapping thermal images (image 1 left, image 2 right)

3.3.4 From single images to image block: The described algorithm for the extraction of tie points between two overlapping images was extended to process the whole image block. In this process, the results from the synchronisation step are required. The images are arranged in strips and to each image an approximate external orientation was assigned. This information is used to find for each image the overlapping images. After detecting the tie points between all overlapping images, the overall tie points are generated by checking which tie point were found in multiple images. At the end, the final tie points can be exported in different data formats containing a point ID, the sub pixel image coordinates and the information of the corresponding images.

\subsection{Orthophoto generation}

The aim of the project was the generation of applied multitemporal orthophotos. Based on the experiences from the tie point extraction and matching the unusual way using a software made for the processing of airborne laser scanning (ALS) data and aerial photos was chosen. The advantage of TerraPhoto (from Terrasolid) is, that external created tie points could be integrated easily. Furthermore, the processing is supported by a digital terrain model (DTM). For this paper, an existing DTM from ALS data was used. It is planned to generate a DTM from image data of the GoPro to dispense with external data. The outlier reduction in section 3.3.3 could not eliminate all mismatches. So these were iteratively improved within the program. In this process also the image orientation parameters and camera parameters can be adjusted gradually. On the basis of tie point ray matches, the adjustment can be 
assessed and controlled. Therefore, it is important that each image has a sufficient number and a good distribution of tie points. Control points are also integrated into the processing. They are mainly used to correct a planar shift of the image block.

\section{RESULTS}

This section will show the results for processing the morning mission and the midday mission. The evening mission had several anomalies to the flight planning whereby more effort is required for the image orientation.

Firstly the results of the tie point extraction will be shown. The next part deals with the results of the orthophoto generation. Additionally, the thermal information from the images was compared to ground measurements obtained to the time of flight.

\subsection{Tie point extraction}

The thresholds for the feature detection were the same for both missions. The following table summarises the results of the tie point extraction.

\begin{tabular}{|l|r|r|}
\hline & Mission 1 & Mission 2 \\
\hline Number of images & 186 & 195 \\
\hline $\begin{array}{l}\text { Average number of } \\
\text { keypoints per image }\end{array}$ & 648 & 1056 \\
\hline Number of final tie points & 16618 & 43185 \\
\hline Processing time & $03: 08 \mathrm{~min}$ & $05: 25 \mathrm{~min}$ \\
\hline
\end{tabular}

Table 2. Results of tie point extraction

For mission 2 (midday) more keypoints and accordingly more tie points were detected compared to mission 1 (morning). This results from a higher image contrast caused by different heating of objects. Advantageous compared to a manually tie point measurement is that in this time an operator could not set so much tie points and that the automatic algorithm finds also tie points in regions with poor image contrast.

For the following orthophoto generation, the high number of tie points in mission 2 was approximately reduced by half, because it could not be handled in TerraPhoto.

\subsection{Orthophoto generation}

In the course of the orthophoto generation the number of tie points was revised again. Table 3 summarises the number of remaining tie points which are the basis for the following image orientation.

\begin{tabular}{|r|r|r|}
\hline & Mission 1 & Mission 2 \\
\hline Number of tie points & 12431 & 9422 \\
\hline Min. tie points per image & 18 & 32 \\
\hline Max. tie points per image & 449 & 309 \\
\hline Average number of tie points & 174 & 119 \\
\hline
\end{tabular}

Table 3. Number of used tie points

Figure 9 shows the orthophotos which were generated for mission 1 (morning) and mission 2 (midday). Comparing both, radiometric differences are obvious. The midday mission is generally brighter and shows a higher image contrast which results from a higher and varying warming of the object surfaces at noon. Furthermore, higher objects like buildings are represented incompletely and show shifts between the strips as well as in neighboring images. This results from the small image format of the thermal camera and the low flight altitude whereby multiple images are necessary to cover a building in whole. To improve the orthophoto concerning this matter, appropriate seamlines have to be defined and building polygons have to be included within creating mosaics.
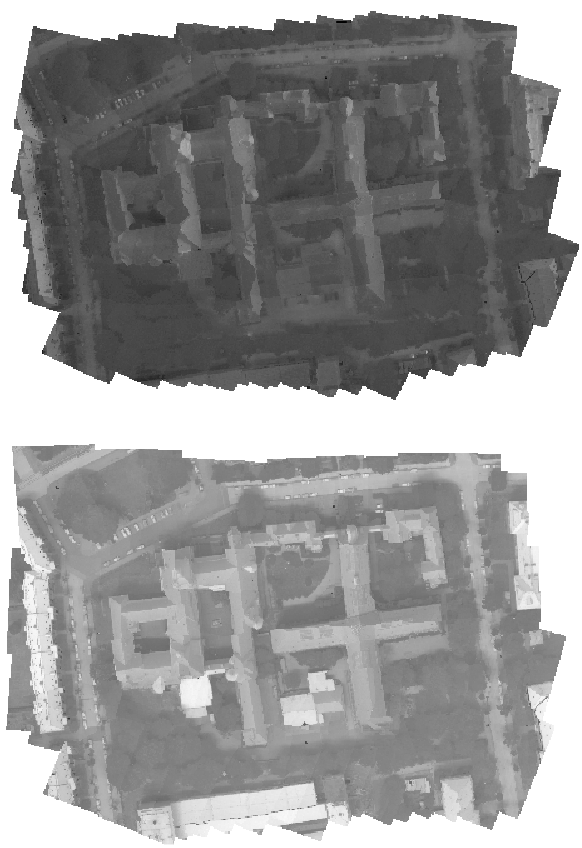

Figure 9. Resulting orthophotos for the morning mission (top) and the midday mission (below)

Concerning the precision of the image orientation, in TerraPhoto no standard deviation of the unit weight can be derived for evaluating the interior precision. The reported averaged control point deviation is up to $4 \mathrm{~cm}$ whereat no differences between height and position are obvious. These values are very optimistic because the control points get large weights during the image orientation and the orthophotos are fixed to it. Another parameter to evaluate the accuracy of the results is the averaged pixel ray mismatch at every tie point which yields with $7.1 \mathrm{~cm}$ (mission 1) and $6.8 \mathrm{~cm}$ (mission 2) very similar values for both missions. But comparing both orthophotos visually, local differences as well as geometric errors are obvious e.g. bends or shifts in roads.

Considering additional check points, a more objective evaluation is possible. The positioning accuracy at four checkpoints along the road, which coordinates were obtained with dGPS, is between $9 \mathrm{~cm}$ and $1.40 \mathrm{~m}$ (mission 1) and between $9 \mathrm{~cm}$ and $42 \mathrm{~cm}$ (mission 2).

These local effects result from the quality of the tie points on the one hand as well as of the number and distribution of the control points on the other hand. To achieve better results, additional control points shall be derived by analysing the RGB data from the GoPro camera. 


\subsection{Comparison of radiation temperatures from thermal data with ground measurements}

Simultaneous measurements on the ground with a second handheld thermal camera (Raytek MiniTemp) were done. This comparison of measurements should give an indicator about the validity of the radiation temperatures measured on the UAV. Different materials (asphalt, concrete, stone) were compared at an emissivity value of 1 . On each surface multiple measurements were done or several values were picked up from the thermal images which were then averaged. Subsequently, the average difference was calculated (reference values are the measurements on the ground). Table 4 shows the results at a glance.

\begin{tabular}{|c|c|}
\hline Mission & $\begin{array}{l}\text { Average difference }[\mathrm{K}] \\
\text { (standard deviation) }\end{array}$ \\
\hline 1 & $-0.5(0.6)$ \\
\hline 2 & $3.9(1.9)$ \\
\hline 3 & $-0.5(0.5)$ \\
\hline
\end{tabular}

Table 4. Comparison of radiation temperatures

As it can be seen from the table, the mission at noon has a significant deviation to the ground measurements. It is believed that the operating temperature of the camera could not be maintained by the movement of the UAV at the midday heat. And thus the measured temperatures are too high. Tests in cooperation with the camera manufactures have to show how to avoid this, e.g. by using a suitable protection shell. It is recommended in any case to realise comparison measurement to confirm the correct operation of the thermal camera.

\section{SUMMARY AND OUTLOOK}

In this paper, an approach was presented which allows the generation of orthophotos from thermal images acquired from an UAV platform. Applying this method, two independent flight missions could be processed and multitemporal orthophotos were generated.

The implemented automatic tie point extraction has potential and the integrated SIFT is well suited for the feature detection and description because it is able to handle the special characteristics of the input data e.g. low image contrast as well as rotation, translation and scale differences due to a more instable platform. But there are still mismatches that have to be eliminated before image orientation. Therefore the parameters have to be optimised and adapted to the respective missions to consider e.g. contrast differences. The idea is to concentrate more on qualitative as on quantitative matches. The synchronisation of the image and the trajectory data had to be realised manually due to a missing common time stamp and the accuracy depends on how well the start image can visually be detected. Because the accuracy influences the success of the tie point extraction as well as of the image orientation, future flight missions should consider this by gathering single images and logging the time stamp for each image already during the flight. However, additional information about the control of the camera (e.g. within a Software Development Kit) from the manufacturer are required.

Currently the RGB image data from the GoPro camera are not involved in data processing. The idea for future work is the photogrammetric analysis of the data to derive additional data e.g. a digital elevation model, building polygons and control points to support the generation of thermal orthophotos or check points for a better evaluation of the exterior precision.

\section{ACKNOWLEDGEMENT}

The authors would like to acknowledge the Fraunhofer Institute for Transportation and Infrastructure Systems IVI for providing the hardware and realising the flight missions. REFERENCES

Bláha, M.; Eisenbeiss, H.; Grimm, D. \& Limpach, P., 2011. Direct georeferencing of uavs. In: Proceedings of the International Conference on Unmanned Aerial Vehicle in Geomatics (UAV-g), Vol. XXXVIII, Zurich, Switzerland.

Eugster, H,. Nebiker, S. (2007): Geo-registration of Video Sequences captured from mini UAVs - Approaches and Accuracy Assessment, The 5th International Symposium on Mobile Mapping Technology, Padua

Fischler, M. A. \& Bolles, R. C., 1981. Random Sample Consensus: A Paradigm for Model Fitting with Applications to Image Analysis and Automated Cartography. Communications of the ACM, Vol. 24(6), pp. 381-395.

Hartmann, W.; Tilch, S.; Eisenbeiss, H. \& Schindler, K., 2012. Determination of the uav position by automatic processing of thermal images. Editor(s): M. Shortis, M. Molenaar, ISPRS Archives - Volume XXXIX-B6, XXII ISPRS Congress, Technical Commission VI, 25 August - 01 September 2012, Melbourne, Australia.

Lowe, D. G., 2004. Distinctive image features from scaleinvariant keypoints. International Journal of Computer Vision, 60 (2), pp. 91-110.

Luhmann, T., Ohm, J., Piechel, J. and Roelfs, T., 2011. Geometric calibration of thermal cameras. Photogrammetrie - Fernerkundung - Geoinformation 2011(1), pp. 5-15.

Meierhold, N.; Spehr, M.; Schilling, A.; Gumhold, S.; Maas, H.-G, 2010. Automatic feature matching between digital images and $2 \mathrm{~d}$ representations of a $3 \mathrm{~d}$ laser scanner point cloud. International Archives of Photogrammetry, Remote Sensing and Spatial Information Science, Volume XXXVIII, Part 5, WG V/3 